\title{
The onset of convection caused by buoyancy during transient heat conduction in deep fluids
}

\begin{abstract}
The onset of convection in a thermal layer generated by transient heat conduction in deep fluid is examined. It is generally accepted that buoyancy driven convection predominates in deep fluids while surface tension driven convection can occur only in very thin layers of liquid. The occurrence of buoyancy convection can be predicted from conventional linear stability analysis for steady-state heat conduction. Its results are summarised in terms of critical Rayleigh numbers. The point of instability in transient heat conduction is, however, less well understood. Its onset in deep fluids is determined by the mode and rate of cooling. In this paper, the judicial application of transient heat conduction equations and a newly defined transient local $\mathrm{Ra}$ with the appropriate boundary conditions has allowed the tracking of the time and spatial development of local hydrodynamic equilibrium to the point of instability. The onset of convection can be predicted from the maximum transient Ra whose values are the same as those previously obtained by linear stability analysis for the same boundary conditions. The critical times and critical depths for stable diffusion in fluids (i.e. without convection) can thus be determined accurately. Agreement with observed values from the literature is very good. The mode and rate of heat conduction are confirmed to be the controlling factors in determining the time of onset of convection.
\end{abstract}

Keyword: Onset of convection; Transient Rayleigh number 